\title{
Class IC antiarrhythmic drug induced atrial flutter: electrocardiographic and electrophysiological findings and their importance for long term outcome after right atrial isthmus ablation
}

\author{
A Nabar, L M Rodriguez, C Timmermans, R van Mechelen, H J J Wellens
}

\begin{abstract}
Objective-To describe the electrocardiographic and electrophysiological findings of new atrial flutter developing in patients taking class IC antiarrhythmic drugs for recurrent atrial fibrillation, and to report the long term results of right atrial isthmus ablation in relation to the ECG pattern of spontaneous atrial flutter.

Design-Retrospective analysis.

Setting-Tertiary care academic hospital.

Patients-24 consecutive patients with atrial fibrillation (age 54 (12) years; 5 female, 19 male) developing atrial flutter while taking propafenone $(n=12)$ or flecainide $(n=12)$.

Results-The ECG was classified as typical $(n=13 ; 54 \%)$ or atypical atrial flutter $(\mathrm{n}=8)$ or coarse atrial fibrillation $(n=3)$. Counterclockwise atrial flutter was the predominant arrhythmia. Acute success after isthmus ablation was similar in patients with typical (12/13) and atypical (8/8) atrial flutter. After long term follow up (13 (6) months, range 6-26 months), continuation of antiarrhythmic drug treatment appeared to result in better control of recurrences of atrial fibrillation in patients with typical atrial flutter $(11 / 13)$ than in those with atypical atrial flutter (4/8), but the difference was not significant. Ablation for coarse atrial fibrillation was unsuccessful.

Conclusions-New atrial flutter developing in patients taking class IC antiarrhythmic drugs for recurrent atrial fibrillation has either typical or atypical flutter wave morphology on ECG. The endocardial activation pattern and the acute results of ablation suggest that the flutter circuit was located in the right atrium and that the isthmus was involved in the re-entry mechanism. There appeared to be better long term control of recurrent atrial fibrillation in patients with typical $(85 \%)$ as compared with atypical atrial flutter $(50 \%)$. Patients developing coarse atrial fibrillation may not be candidates for this strategy.

(Heart 2001;85:424-429)
\end{abstract}

Keywords: atrial flutter; antiarrhythmic agents; fibrillation; ablation

The architecture of the right atrium provides natural obstacles or barriers promoting the development of atrial flutter. ${ }^{1}$ Indeed, in $80-85 \%$ of the cases, the macro re-entrant flutter circuit is located in the right atrium. ${ }^{2}$ In comparison, atrial fibrillation may be based on either a "mother" macro re-entrant circuit with "daughter" waves, random re-entry, or rapid focal activity. " 4 "Organisation" of atrial fibrillation to flutter requires prolongation of the re-entrant wavelength, ${ }^{5}$ thereby resulting in a single macro re-entrant loop. Antiarrhythmic drugs promote this organisation, ${ }^{6}$ probably by slowing isthmus conduction ${ }^{7}$ and limiting transverse conduction across the crista terminalis. The incidence of atrial flutter in patients taking class IC antiarrhythmic drugs for recurrent atrial fibrillation is estimated to range from $3.5-20 \%,{ }^{8}$ but probably depends on the frequency with which ECGs are recorded.

Both "classical" and atypical electrocardiographic and atrial activation patterns have been described in patients with atrial fibrillation developing atrial flutter while taking class IC antiarrhythmic drugs. ${ }^{9}{ }^{10}$ In these patients, combined treatment with isthmus ablation and antiarrhythmic drugs has been reported to prevent or reduce the recurrences of atrial fibrilla- tion. ${ }^{6910}$ The value of this approach needs to be determined by results obtained after long term follow up.

We recently reported on the development of atrial flutter in patients taking class IC antiarrhythmic drugs such as propafenone or flecainide for recurrent episodes of atrial fibrillation. ${ }^{9}$ Our aim in the present study was first, to describe the ECG characteristics and electrophysiological findings during radiofrequency ablation of this new atrial flutter, and second, to report on the long term results of right atrial isthmus ablation in relation to the ECG pattern found during a clinical episode of atrial flutter.

\section{Methods}

STUDY POPULATION

Twenty four consecutive patients (mean (SD) age 54 (12) years; 5 female, 19 male) -who had been symptomatic with recurrent and disabling episodes of atrial fibrillation for 8 (9) years (range 3 months to 30 years), and who developed a new atrial flutter while taking a class IC antiarrhythmic drug - underwent an electrophysiological study and a radiofrequency ablation procedure. 


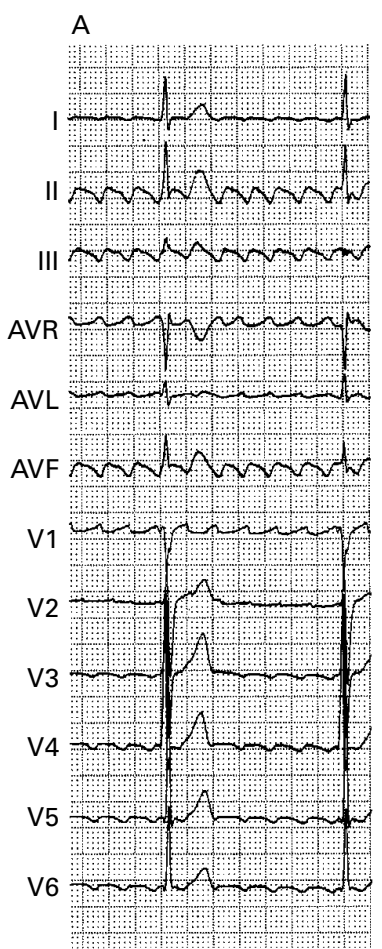

B

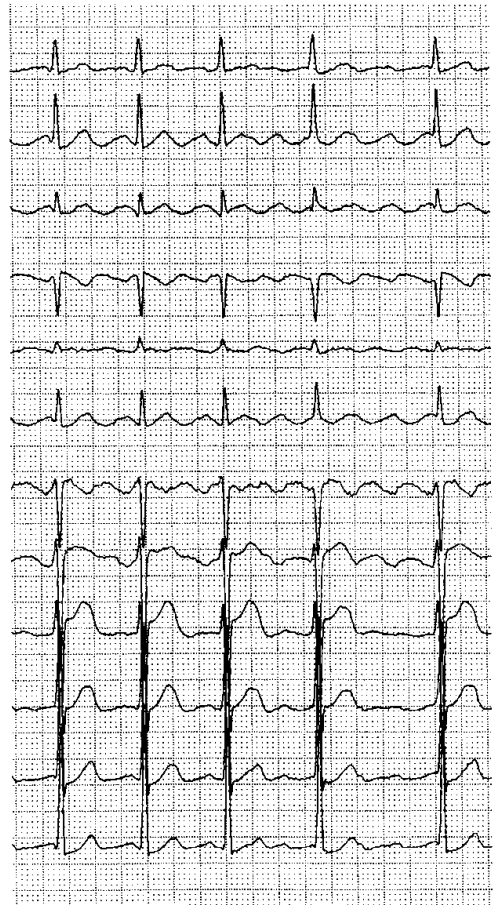

C

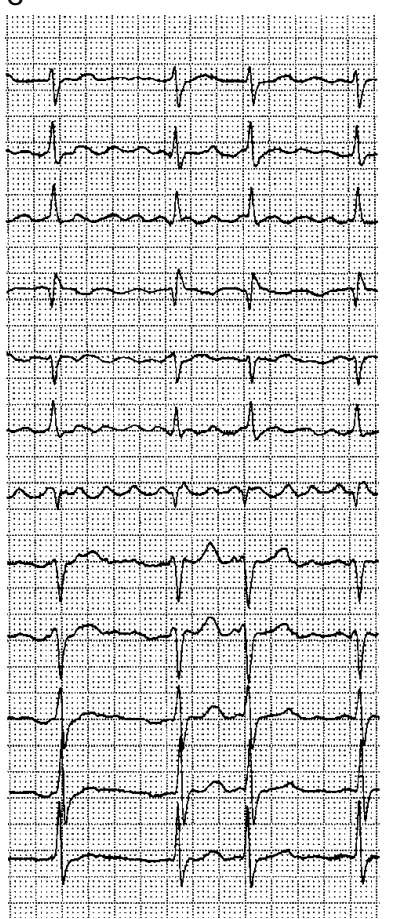

Figure 1 The 12 lead ECG shows: (A) typical atrial flutter with regular and sharp negative atrial deflections in the inferior leads and positive ones in lead V1; (B) atypical atrial flutter with positive and rounded atrial deflections in the inferior leads - the deflections in lead V1 are negative and rounded; and (C) coarse atrial fibrillation with varying intervals and morphology of the atrial deflections.

Structural heart disease was present in seven patients, and the mean left atrial diameter was $4.6(0.4) \mathrm{cm}$. Atrial fibrillation was paroxysmal in nine patients and persistent in 15 . Prolonged periods of sinus rhythm could not be maintained in spite of the use of a mean of three antiarrhythmic drugs/patient (SD 1.6; range 1-6). Three patients had previously received an atrial defibrillator (Metrix, Atrioverter, InControl Inc, Redmond, Washington, USA) and one a dual chamber pacemaker for sick sinus syndrome. Atrial flutter was never documented before the use of class IC antiarrhythmic drugs. An equal number of patients was taking propafenone (450-900 mg/day) or flecainide (100-400 mg/day) when organisation to atrial flutter was observed. Concomitant atrioventricular node blocking drugs were used in most patients, to avoid a $1: 1$ ventricular response during an episode of atrial flutter.

\section{ELECTROCARDIOGRAM}

A 12 lead ECG recorded during the clinical episode of atrial flutter was classified in retrospect by one of the investigators (HW), unaware of the findings during the electrophysiological study. Typical atrial flutter was defined as the presence of regular flutter waves showing negative deflections (or predominantly negative with a terminal positive part) in the inferior leads, and an entirely or predominantly positive flutter wave in lead V1 (fig 1A). ${ }^{2}$ Atrial flutter with uniform and regular flutter waves but another morphology-either in the inferior leads or in lead V1, or both-was classified as atypical (fig 1B).
ELECTROPHYSIOLOGICAL STUDY AND RADIOFREQUENCY ABLATION PROCEDURE An electrophysiological study and radiofrequency ablation were performed after informed consent and with the patient in a postabsorptive state. The activation pattern of the right atrium was recorded from a duodecapolar Halo catheter (Cordis-Webster, Baldwin Park, California, USA), and preferably a multipolar catheter (Cordis-Webster) was used to record activation of the coronary sinus. When possible, bidirectional conduction across the isthmus was evaluated before ablation. Induction of atrial flutter was attempted with maximal 3 atrial extrastimuli and incremental atrial pacing. We refrained from performing entrainment mapping because of the induction of sustained atrial fibrillation earlier in this series. If sustained atrial fibrillation was inadvertently induced, sinus rhythm was restored before continuing the procedure. Ablation of the right atrial isthmus was performed using an anatomical approach. ${ }^{9}{ }^{11}$

ACUTE PROCEDURAL RESULT

Success of isthmus ablation was judged by non-inducibility of atrial flutter and bidirectional isthmus conduction block, confirmed under isoprenaline (isoproterenol) infusion. ${ }^{12}$ Non-inducibility in the absence of complete bidirectional isthmus conduction block was considered a partial success. The procedure had failed if the flutter remained inducible.

FOLLOW UP PROTOCOL

Patients were advised to continue taking propafenone or flecainide. Follow up assess- 
Table 1 Findings during electrophysiological study

\begin{tabular}{llll}
\hline & $\begin{array}{l}\text { Typical } \\
\text { atrial flutter } \\
(n=13)\end{array}$ & $\begin{array}{l}\text { Atypical } \\
\text { atrial futter } \\
(n=8)\end{array}$ & $\begin{array}{l}\text { Coarse atrial } \\
\text { fibrillation } \\
(n=3)\end{array}$ \\
\hline $\begin{array}{l}\text { Atrial flutter inducibility (n) } \\
\quad \text { Continuously present }\end{array}$ & 4 & 2 & 3 \\
$\quad$ Induced & 3 & 3 & 0 \\
$\quad$ Non-inducible & 6 & 3 & 0 \\
Right atrial activation during atrial flutter $(\mathrm{n})$ & $(\mathrm{n}=7)$ & $(\mathrm{n}=5)$ & $(\mathrm{n}=3)$ \\
$\quad$ Counterclockwise & 7 & 4 & 3 \\
Clockwise & 0 & 1 & 0 \\
Left atrial response during atrial flutter $(\mathrm{n})$ & $(\mathrm{n}=6)$ & $(\mathrm{n}=5)$ & $(\mathrm{n}=1)$ \\
$\quad \begin{array}{l}1: 1 \\
\text { Atrial fibrillation }\end{array}$ & 6 & 5 & 0 \\
& 0 & 0 & 1 \\
\hline
\end{tabular}

ment included a clinical history, ECG, and Holter recordings at eight weeks and then at three month intervals.

Patients having a documented recurrence of atrial flutter were advised to undergo reablation. Patients who had no recurrences of atrial fibrillation, asymptomatic recurrences (detected by Holter, Atrioverter, or pacemaker device alone), or symptomatic recurrences that were essentially paroxysmal and had responded to maintenance antiarrhythmic drugs at the time of last follow up were considered to have improved clinically and therefore to be well controlled. These patients were taking either propafenone or flecainide, and occasionally a class III antiarrhythmic drug or a calcium antagonist was added to the regimen. Patients who continued to have frequent and symptomatic recurrences of atrial fibrillationincluding persistent episodes requiring cardioversion (by external shock or Atrioverter) - or who remained symptomatic with recurrences at the latest follow up despite alterations in the antiarrhythmic drug regimen, were considered to be inadequately controlled. These patients had not profited from isthmus ablation.
STATISTICAL ANALYSIS

Results are expressed as mean (SD), with ranges. Mean values were compared by one way analysis of variance, and $\chi^{2}$ tests were used for assessing homogeneity in contingency tables. Results were considered to be significant at $\mathrm{p}<0.05$.

\section{Results}

12 LEAD ECG DURING A CLINICAL EPISODE

Thirteen of 24 patients (54\%) had typical atrial flutter. In eight other patients, the flutter wave was atypical in the inferior leads or in lead V1. The mean flutter cycle length was 268 (34) ms (range 220-360 ms). The atrioventricular conduction ratio was variable in nine patients, fixed at $2: 1$ in 11 , and fixed at $1: 1$ in one (flutter rate $360 \mathrm{~ms}$ ). The remaining three patients had small differences in the intervals and configuration of atrial deflections and they were classified as having fibrilloflutter or coarse atrial fibrillation (fig 1C).

\section{FINDINGS DURING THE ELECTROPHYSIOLOGICAL} STUDY

Table 1 summarises the findings during the electrophysiological studies. Atrial flutter could be recorded during the study in 12 of 21 patients, but was not inducible in nine patients. There was no difference with regard to inducibility in patients with typical or atypical atrial flutter. In the three patients later classified as having coarse atrial fibrillation, this rhythm was present during most of the procedure.

The rotation of atrial flutter was counterclockwise in 11 of 12 patients (fig $2 \mathrm{~A}$ ). In the three patients with atypical atrial flutter, the induced flutter was electrocardiographically similar to the clinical flutter and of counterclockwise rotation in one; electrocardiographically different with clockwise rotation in one
A

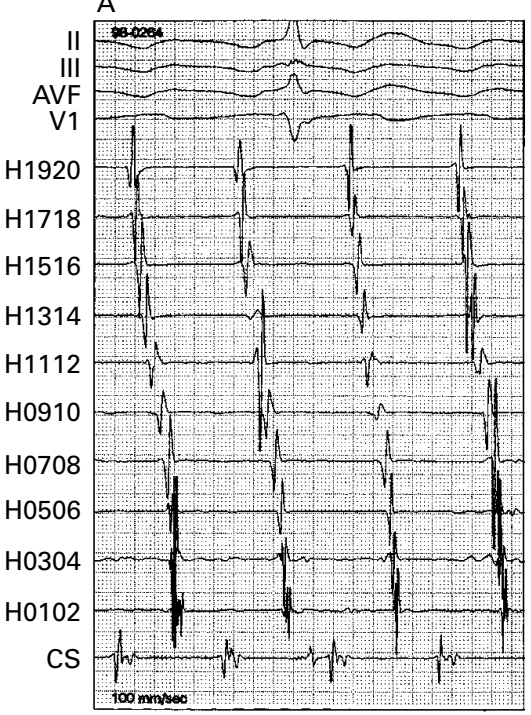

B

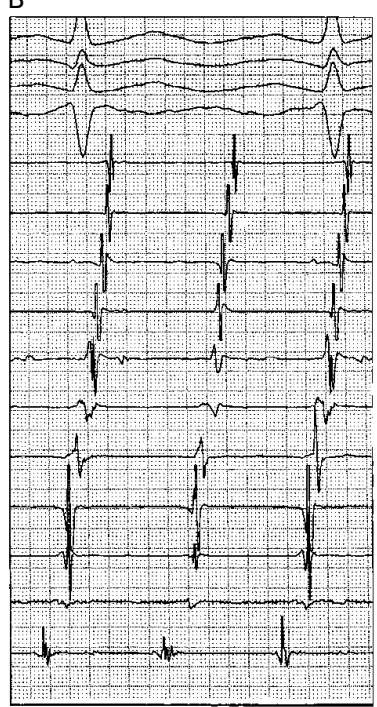

C

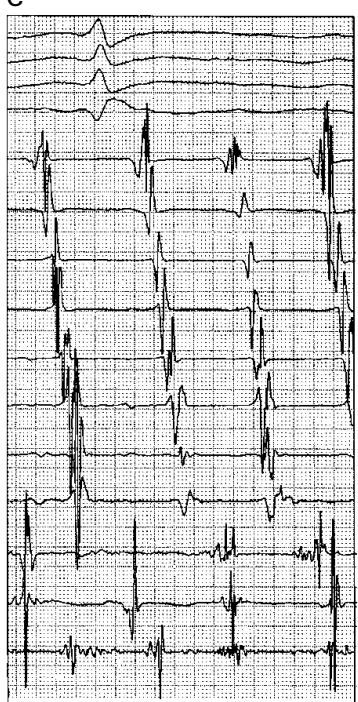

Figure 2 Intra-atrial activation pattern of the same three patients shown in fig 1. (A) Counterclockwise rotation of the atrial flutter (260 ms). (B) Clockwise rotation of the atrial flutter (310 ms). (C) Directionally stable right atrial activation sequence (that is, counterclockwise), but with beat to beat variations in the cycle length. Note that the electrograms from coronary sinus suggest a 1:1 left atrial response in all three patients. Surface ECG leads II, III, AVF, and $V 1$ are shown. The intracardiac electrograms include bipoles of the duodecapolar (Halo) catheter positioned around the tricuspid annulus (H0102-1920) and the bipole located at the coronary sinus ostium (CS). 


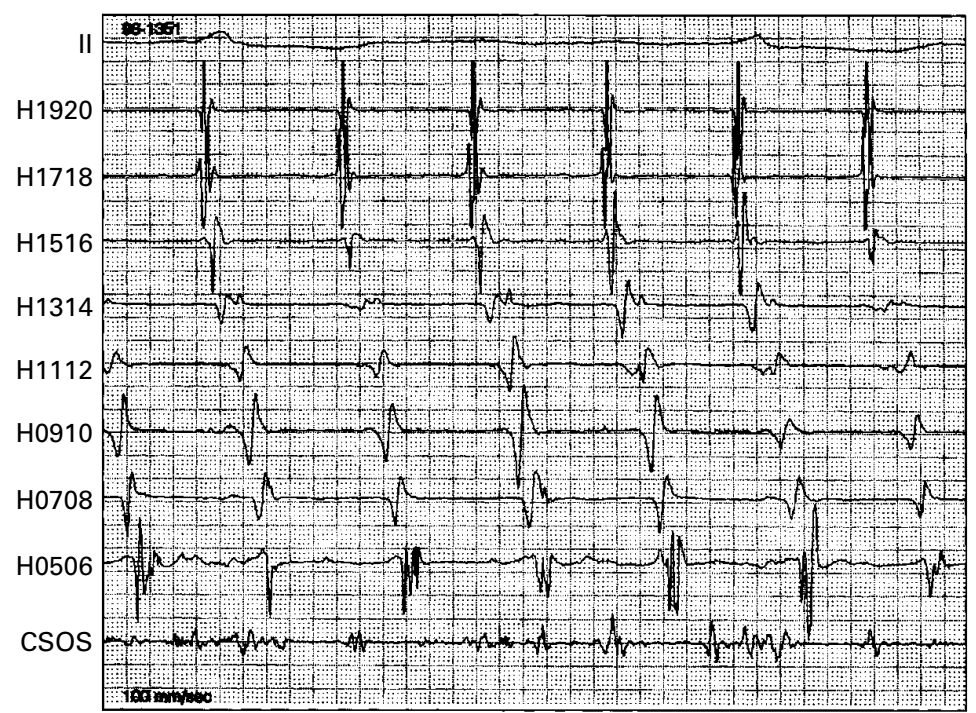

Figure 3 An example of coarse atrial fibrillation. Note that the right atrial endocardial activation shows counterclockwise rotation and cycle length stability. However, the electrograms from the coronary sinus show left atrial fibrillation. H0506-1920 and CS represent intracardiac electrograms from bipoles of Halo and coronary sinus catheter.

Table 2 Long term results following right atrial isthmus ablation in the 24 patients

\begin{tabular}{|c|c|c|c|}
\hline & $\begin{array}{l}\text { Typical atrial } \\
\text { flutter }(n=13)\end{array}$ & $\begin{array}{l}\text { Atypical atrial } \\
\text { flutter }(n=8)\end{array}$ & $\begin{array}{l}\text { Coarse atrial } \\
\text { fibrillation }(n=3)\end{array}$ \\
\hline \multicolumn{4}{|l|}{ Atrial flutter ablation } \\
\hline Success & 11 & 7 & \\
\hline Partial success & 1 & 1 & \\
\hline Failure & 1 & 0 & 3 \\
\hline Duration of follow up (months) & $8-21$ & $6-26$ & $6-10$ \\
\hline Atrial flutter recurrences & 3 & 1 & \\
\hline \multicolumn{4}{|l|}{ Atrial fibrillation recurrences } \\
\hline Well controlled & 11 & 4 & 0 \\
\hline Not controlled & 2 & 4 & 3 \\
\hline
\end{tabular}

(fig 2B); and had two ECG morphologies in the third, one of which was identical to the clinical arrhythmia and the other different from it, both with counterclockwise rotation.

As expected, and despite directional stability (that is, counterclockwise rotation) (fig 2C), patients with coarse atrial fibrillation (two of the three) had beat to beat variation in atrial cycle length. The atrial cycle length was stable in the remaining patient.

During the arrhythmia, a recording from the coronary sinus was available in 12 of 15 patients. This revealed a 1:1 left atrial response in 11 and left atrial fibrillation in one patient with coarse atrial fibrillation (fig 3 ). Coronary sinus activation was recorded by a multipolar catheter in only five of the above 11 patients. In four of these, who had counterclockwise atrial flutter, the activation sequence was as follows: proximal to distal $(n=1)$, distal to proximal $(n=2)$, or simultaneous $(n=1)$. Coronary sinus activation was proximal to distal in one patient who had clockwise atrial flutter.

ATRIAL FIBRILLATION DURING THE ABLATION PROCEDURE

Sustained atrial fibrillation was precipitated inadvertently in eight of 21 patients, including five patients with typical atrial flutter and three with atypical flutter. All three patients with coarse atrial fibrillation showed disorganisation of right atrial activation (absence of repetitive activation pattern along the Halo catheter) on at least one occasion.

Sinus rhythm was restored by external $(\mathrm{n}=5)$ or internal $(\mathrm{n}=2)$ electrical cardioversion, intravenous flecainide $(n=1)$, or overpacing $(n=1)$. In the remaining two patients, spontaneous reversion to atrial flutter was observed.

\section{ACUTE PROCEDURAL RESULT}

A linear ablation of the right atrial isthmus was performed during coronary sinus pacing $(n=13)$, atrial flutter $(n=6)$, or both rhythms $(\mathrm{n}=4)$. Because of recurrent spontaneous induction of atrial fibrillation, ablation was performed during sinus rhythm (without coronary sinus pacing) in one patient. The results of ablation are included in table 2 .

Flutter ablation ( $\mathrm{n}=21)$ was judged successful in 18 patients, partially successful in two (one each with typical and atypical atrial flutter), and failed in one (typical atrial flutter). In the three patients with coarse atrial fibrillation, the arrhythmia persisted despite ablation of both isthmuses (tricuspid annulus to inferior vena cava and tricuspid annulus to coronary sinus) in two patients, and it was not inducible after a bidirectional isthmus conduction block in the third. Thus, in contrast with the results in the patients with coarse atrial fibrillation, the acute success rate of isthmus ablation in patients with typical (12/13) and atypical (8/8) atrial flutter was similar and high.

LONG TERM FOLLOW UP RESULTS

Follow up data were available over a mean of 13 (6) months (range 6-26 months). After an initially successful ablation, four patients (three with typical and one with atypical atrial flutter) had a recurrence of atrial flutter after a mean of 9 (4) months (range 3-15 months). In these four patients, acute success was judged by non-inducibility alone $(n=1)$, noninducibility and unidirectional isthmus conduction block $(\mathrm{n}=1)$, and non-inducibility and bidirectional isthmus conduction block $(n=2)$. All four patients underwent successful reablation, with confirmation of bidirectional isthmus conduction block under isoprenaline infusion, and were free of further atrial flutter recurrences at the latest follow up.

Of the 13 patients who underwent ablation for typical atrial flutter, recurrences of atrial fibrillation were prevented in 11. Class IC antiarrhythmic drugs were stopped in two patients, while nine continued taking them, either alone $(n=7)$ or in combination with sotalol $(n=2)$. Recurrent episodes of atrial fibrillation occurred in two patients, including the patient with operated Ebstein's anomaly who had an unsuccessful atrial flutter ablation. The other patient had recurrences of atrial fibrillation in spite of the addition of amiodarone to flecainide, and after successful isthmus reablation for recurrence of atrial flutter.

After ablation of atypical atrial flutter $(n=8$ patients), four patients showed a reduction in atrial fibrillation recurrences while taking class IC drugs alone. The frequency of atrial fibrillation recurrence did not change in the other 
four patients. Among the latter, two received recurrent therapeutic shocks from an Atrioverter device and one underwent elective external cardioversion on four occasions. Thus the combination of isthmus ablation and antiarrhythmic drug treatment resulted in better control of atrial fibrillation recurrence in patients with typical compared with atypical atrial flutter $(85 \% v 50 \%)$. Because of the small numbers of patients, this difference did not reach significance.

Not surprisingly, none of the three patients with coarse atrial fibrillation improved, and they continued to have frequent recurrences of atrial fibrillation. One patient developed chronic persistent atrial fibrillation (table 2).

\section{Discussion}

CLASS IC ANTIARRHYTHMIC DRUG INDUCED ATRIAL FLUTTER

In nearly half the patients in the present study, the ECG morphology of the new atrial flutter was typical in type; in the remainder it was classified as atypical atrial flutter or coarse atrial fibrillation. The mean flutter rate (268 (34 ms)) was slow because of the class IC antiarrhythmic drug treatment. With regard to the right atrial activation pattern, no differences were found between typical and atypical atrial flutter. The flutter circuit was localised to the right atrium and showed a counterclockwise rotation in most patients. In the majority, the left atrium responded to the right atrial re-entrant circuit in a $1: 1$ fashion, but with a variable sequence of coronary sinus activation. This finding is compatible with the left atrium being a bystander and not being essential to the atrial flutter circuit. ${ }^{2}$ The importance of the left atrial activation sequence in determining the ECG polarity of the flutter wave was shown in a canine model of atrial flutter. ${ }^{13}$ In the present study, the few multipolar recordings from the coronary sinus are insufficient to suggest global left atrial activation and therefore it remains speculative whether the differences in coronary sinus (left atrial) activation are responsible for the observed differences in flutter wave morphology. Lai and colleagues have pointed out the lack of correlation between the direction of rotation of atrial flutter and the polarity in the inferior leads. ${ }^{14}$

RADIOFREQUENCY ABLATION OF ATRIAL FLUTTER A better outcome has been reported after ablation of typical atrial flutter that develops in patients being treated with amiodarone for recurrent atrial fibrillation. ${ }^{6}$ In the present study, after isthmus ablation, non-inducibility of atrial flutter was seen in $92 \%$ of patients with typical atrial flutter and in all patients with atypical atrial flutter. The endocardial activation pattern and the acute results of ablation seem to suggest that the right atrial isthmus is involved in the re-entry mechanism in both typical and atypical atrial flutter. This finding - particularly in patients with atypical atrial flutter-has important clinical implications, as such patients could still be candidates for isthmus ablation. Chan and colleagues have recently reported isthmus dependent atrial flutter in postoperative intra-atrial re-entrant tachycardia despite lack of the classic sawtooth flutter waves on the surface ECG. ${ }^{15}$

The long term improvement in control of atrial fibrillation recurrence was around $85 \%$ in patients with typical atrial flutter, compared with around $50 \%$ in those with atypical flutter. This difference, although suggesting improved results with typical atrial flutter, was not significant because of the small number of patients available for study.

COARSE ATRIAL FIBRILLATION AFTER CLASS IC ANTIARRHYTHMIC DRUG TREATMENT

Three patients with coarse atrial fibrillation underwent isthmus ablation. All three showed a stable counterclockwise right atrial activation pattern. Beat to beat cycle length variation was present in two of them. Even after ablating both the right atrial isthmuses, non-inducibility could not be achieved in two patients. Atrial fibrillation recurred frequently in all three. These data suggest that when coarse atrial fibrillation is present on the ECG and the right atrial electrograms show cycle length variations, the presence of a stable circular right atrial activation pattern alone may not be sufficient to make these patients candidates for isthmus ablation. In fact, these results prompted us to exclude such patients from further study. However, mapping by the non-contact endocardial mapping system could help to determine the beat to beat characteristics of the right atrial trajectory of this arrhythmia and the role of the isthmus. Conventional entrainment studies evaluating isthmus dependency would be difficult in view of the changing ECG morphology, cycle length variability, and risk of disorganising the arrhythmia.

\section{STUDY LIMITATIONS}

Nearly half the cases of atrial flutter had an atypical flutter wave morphology. In the absence of detailed mapping from atrial septum and left atrium, the reasons for differences in ECG morphology could not be determined.

In nine of the 21 patients (around $40 \%$ ), atrial flutter could not be induced. In these patients with non-inducible but clinically documented atrial flutter, complete bidirectional isthmus conduction block had to be accepted as a procedural end point.

In the absence of entrainment studies, the location of the atrial flutter circuit and its isthmus dependence could not be determined precisely. Non-inducibility of atrial flutter and precipitation of sustained atrial fibrillation early in the series prevented us from doing entrainment mapping.

It is possible that induced atrial flutter may be different from spontaneous atrial flutter, as seen in two patients with induced atypical atrial flutter.

Another important limitation was the small cohort of patients available.

Except in patients with an implanted device, the reported frequency of atrial fibrillation recurrence during follow up is at best an 
estimate. However, the frequency of symptomatic atrial fibrillation recurrence requiring treatment is directly relevant to the patient.

CONCLUSIONS

Our study showed that both typical and atypical ECG morphologies are seen in patients with atrial fibrillation who develop atrial flutter while receiving class IC antiarrhythmic drug treatment. The endocardial activation pattern and the acute results of ablation suggest that the atrial flutter circuit was located in the right atrium and that the isthmus was involved in the re-entry mechanism. Although the difference was not significant, long term control of atrial fibrillation recurrence appeared to be easier in patients with typical atrial flutter than in those with atypical flutter. Patients developing coarse atrial fibrillation may not be candidates for ablation treatment.

AN is supported by the Wijnand M Pon Foundation, Leusden, Netherlands. Presented at the 21 st Annual scientific sessions of the North American Society of Pacing and Electrophysiology, and published in abstract form (Pacing Clin Electrophysiol 2000;23(part II):743).

1 Cabrera JA, Sanchez-Quintana D, Ho SY, et al. The architecture of the atrial musculature between the orifice of the inferior vena caval vein and the tricuspid valve: the anatomy of the isthmus. I Cardiovasc Electrophysiol 1998;9:1186-95

2 Cosío FG, Arribas F, Lopez-Gil M, et al. Endocardial catheter mapping of atrial arrhythmias. In: Shenasa $M$ Borggrefe M, Breithardt G, eds. Cardiac mapping. Mount Kisco, NY: Futura, 1993:443-59.

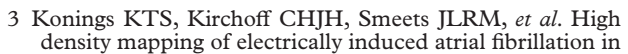
density mapping of electrically induced
humans. Circulation 1994;89:1665-80.

4 Jaï P, Haïssaguerre M, Shah DC, et al. A focal source of atrial fibrillation treated by discrete radiofrequency ablation. Circulation 1997;95:572-6.

5 Matsuo K, Kumagai K, Annoura M, et al. Mechanism of antiarrhythmic effects of class Ic drugs in paroxysmal atrial fibrillation in man. Cardiology 1998;89:119-23.

6 Huang DT, Monahan KM, Zimetbaum P, et al. Hybrid pharmacologic and ablative therapy: a novel and effective
approach for the management of atrial fibrillation. $\mathcal{F}$ Cardiovasc Electrophysiol 1998;9:462-9.

7 Yamashita T, Inoue $\mathrm{H}$, Nozaki A, et al. Role of anisotropy in determining the selective action of antiarrhythmics in atrial determining the selective action of antiarrhythmics
flutter in the dog. Cardiovasc Res 1992;26:244-9.

8 Falk RH. Proarrhythmic responses to atrial antiarrhythmic therapy. In: Falk RH, Podrid PJ, eds. Atrial fibrillation: mechanisms and management. NY: Raven Press, 1992:283305.

9 Nabar A, Rodriguez LM, Timmermans C, et al. Radiofrequency ablation of "class IC atrial flutter" in patients with resistant atrial fibrillation. Am f Cardiol 1999;83:785-7.

10 Schumacher B, Jung W, Lewalter T, et al. Radiofrequency ablation of atrial flutter due to administration of class IC antiarrhythmic drugs for atrial fibrillation. Am $\mathcal{F}$ Cardiol 1999;83:710-13.

11 Poty H, Saoudi N, Nair M, et al. Radiofrequency catheter ablation of atrial flutter: further insights into the various types of isthmus block: application to ablation during sinus rhythm. Circulation 1996;94:3204-13.

12 Nabar A, Rodriguez LM, Timmermans C, et al. Isoproterenol to evaluate resumption of conduction following right enol to evaluate resumption of conduction following right
atrial isthmus ablation in type I atrial flutter. Circulation atrial isthmus ablatio

13 Okumura K, Plumb VJ, Pagé PL, et al. Atrial activation sequence during atrial flutter in the canine pericarditis model and its effects on the polarity of the flutter wave in the electrocardiogram. F Am Coll Cardiol 1991;17:509-18.

4 Lai LP, Lin JL, Lin LJ, et al. New electrocardiographic criteria for the differentiation between counterclockwise and clockwise atrial flutter: correlation with electrophysiological study and radiofrequency catheter ablation. Heart 1998;80:80-5.

15 Chan DP, Van Hare GF, Mackall JA, et al. Importance of atrial flutter isthmus in postoperative intra-atrial reentrant tachycardia. Circulation 2000;102:1283-9.

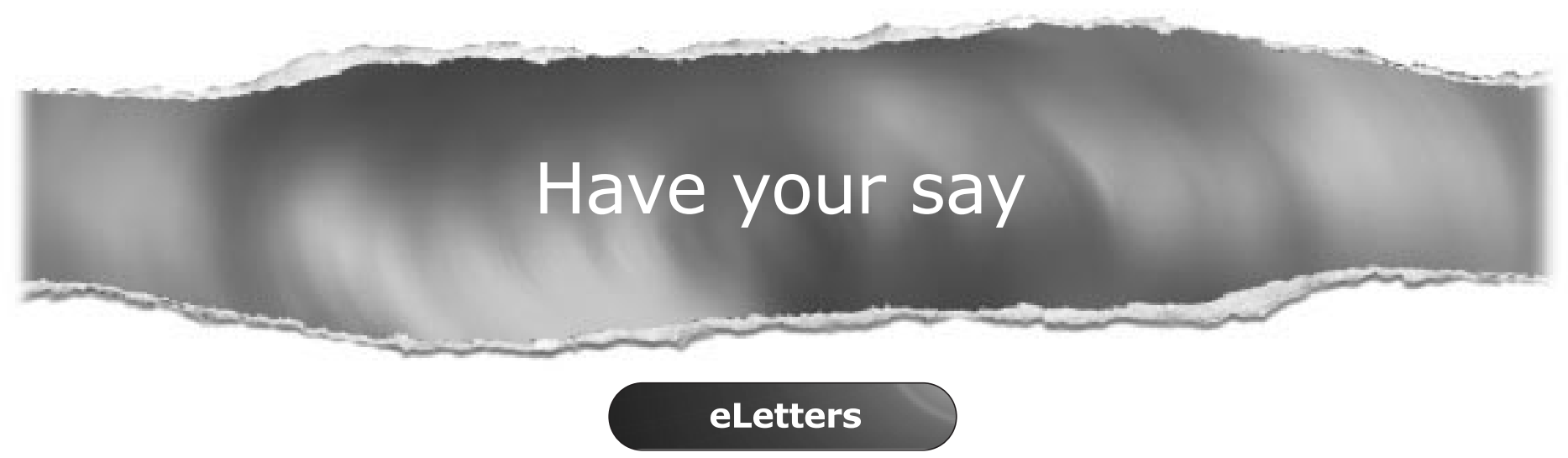

If you wish to comment on any article published in Heart you can send an eLetter using the eLetters link at the beginning of each article. Your response will be posted on Heart online within a few days of receipt (subject to editorial screening).

www.heartjnl.com 